\title{
When menopause takes the sleep away
}

\author{
C. Pinto Ferreira $^{1 *}$, I. Pinto ${ }^{1}$, C. Pereira ${ }^{1}$, R. Borralho ${ }^{1}$, M.J. Avelino ${ }^{1}$
}

${ }^{1}$ Psychiatric Hospital Centre of Lisbon, Portugal

*Corresponding author: catarinaferreira@chpl.min-saude.pt

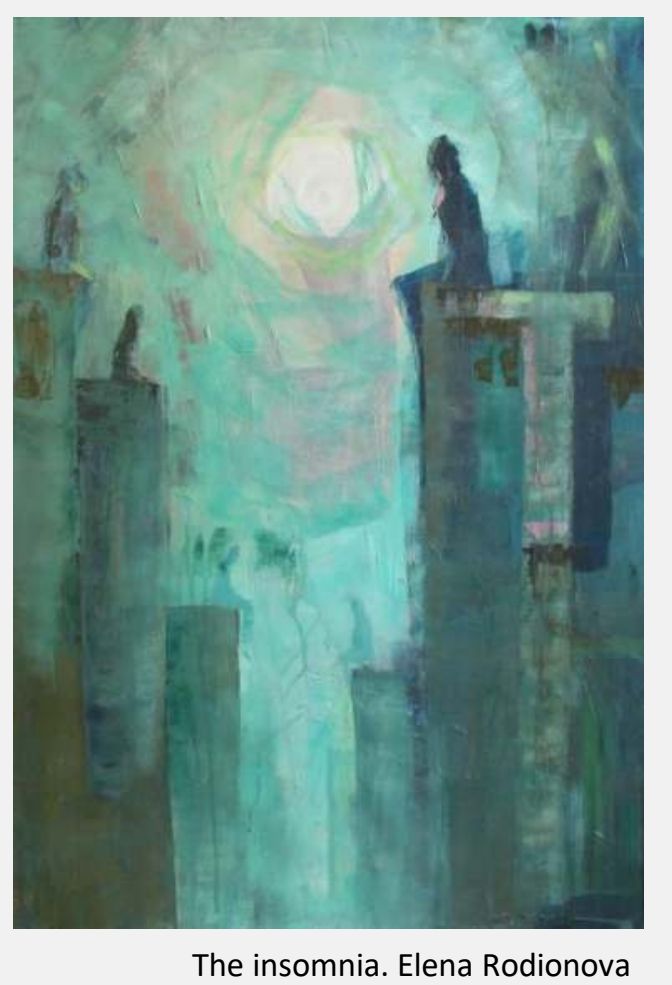

\section{Background:}

Menopausal transition is associated with fluctuating hormone levels which predisposes to several physical and psychological symptoms. Nearly half of women in perimenopause period experience sleep difficulties, with one third of them experiencing insomnia starting in menopause, which may have a significant negative impact in women's quality of life.

\section{Aims:}

The aim of this study is to systematically review the current scientific literature regarding sleep disorders in menopause and the available therapeutic options.

\section{Methods:}

The authors used the search engine PubMed $\AA$ and selected all the articles published in English in the last ten years with the words "sleep disorders" and "menopause" in the title and/or abstract.

\section{Results:}

Hot flashes (HF's) are one of the main causes of sleep disturbances in menopause and are present in up to $80 \%$ of perimenopausal women. They seem to be strongly correlated with poor sleep quality, causing frequent awakenings measured by polysomnography.

Studies have shown that the lower the estrogen and the higher the FSH (follicle-stimulating hormone) levels, the worse is the quality of sleep.

Insomnia in menopause is associated with other psychiatric conditions like depression and anxiety states, predisposing also to physical illness, namely due to autonomic changes.

Treatment options include sleep hygiene, hormonotherapy, antidepressants and cognitive behavioural treatment.

\section{Conclusions:}

Sleep disorders are frequent during perimenopause and have a negative impact in women's quality of life, sometimes leading to psychiatric conditions like depression. Prompt treatment must be provided and given the multifactorial nature of insomnia, an individualized therapeutic strategy must be offered. 